\title{
Percepção dos familiares acerca do grupo de apoio realizado em uma unidade de internação psiquiátrica
}

\author{
Perception of family members regarding the support group conducted in a \\ psychiatric intervention ward \\ Percepción de los familiares acerca del grupo de apoyo realizado en una \\ unidad de internación psiquiátrica
}

\section{Maria de Lourdes Custódio Duarte Juliana de Carvalho ${ }^{b}$ Vivian Brentano ${ }^{c}$}

\section{Como citar este artigo:}

Duarte MLC, Carvalho J, Brentano V.

Percepção dos familiares acerca do grupo de apoio realizado em uma unidade de internação psiquiátrica. Rev Gaúcha Enferm. 2018;39:e2017-0115. doi: https:// doi.org/10.1590/1983-1447.2018.20170115 .
Universidade Federal do Rio Grande do Sul (UFRGS), Escola de Enfermagem, Departamento de Assistência e Orientação Profissional. Porto Alegre, Rio Grande do Sul, Brasil.

b Universidade Federal do Rio Grande do Sul (UFRGS), Escola de Enfermagem, Programa de Pós- Graduação em Enfermagem. Porto Alegre, Rio Grande do Sul, Brasil.

Hospital de Clínicas de Porto Alegre (HCPA), Residência Integrada Multiprofissional em Saúde. Porto Alegre, Rio Grande do Sul, Brasil.

\section{RESUMO}

Objetivo: Objetiva analisar a percepção de familiares de pacientes com sofrimento psíquico acerca do grupo de apoio em uma internação psiquiátrica.

Método: Pesquisa com abordagem qualitativa, exploratória, descritiva, realizada em hospital geral do Rio Grande do Sul com dez familiares que participavam de grupo de apoio semanal. A coleta de dados ocorreu no mês de outubro de 2016 através de entrevistas semiestruturadas. Empregou-se a análise de conteúdo temática para tratamento dos dados, na qual emergiu a categoria: Percepção dos familiares sobre o grupo de apoio.

Resultados: Os familiares percebiam o grupo como um espaço de apoio e fortalecimento, de escuta e trocas entre os integrantes, de informação sobre a doença e tratamento e de segurança e inserção da família no tratamento.

Considerações finais: Portanto, o grupo de apoio pode ser entendido como uma ação estratégica de cuidado ao familiar, repercutindo na sua vida e no tratamento de quem está internado.

Palavras-chave: Enfermagem. Saúde mental. Serviços de saúde. Grupos de autoajuda.

\section{ABSTRACT}

Objective: To analyze the perception of relatives of patients with mental disorders about the support group in a psychiatric hospitalization ward.

Method: This is a research with a qualitative, exploratory and descriptive approach, performed at a General Hospital in Rio Grande do Sul with ten relatives of patients who had been participating in a weekly support group. Data collection took place in October 2016, through semi-structured interviews. A thematic content analysis was used for the treatment of data, whence emerged the category: Perception of family members about the support group.

Results: The relatives perceive the group as a space that gives them strength and support, allowing for listening and experience exchange among its members, giving information on the disease and treatment, safety, and inserting the family in the treatment.

Final considerations: The support group can be understood as a strategic action of caring for the family, affecting their lives and the treatment of those who are hospitalized.

Keywords: Nursing. Mental health. Health services. Self-help groups.

\section{RESUMEN}

Objetivo: Analizar la percepción de familiares de pacientes con trastorno mental acerca del grupo de apoyo en una internación psiquiátrica.

Método: Investigación con abordaje cualitativo, exploratorio y descriptivo, realizada en el hospital General de Rio Grande do Sul, con diez familiares que participaban en un grupo de apoyo semanal. La recolección de datos ocurrió en el mes de octubre de 2016 por medio de entrevistas semiestructuradas. Se empleó al análisis temático de contenido para tratar a los datos, y emergió la categoría: Percepción de los familiares sobre el grupo de apoyo.

Resultados: Los familiares perciben al grupo como un espacio de apoyo y fortalecimiento, de escucha e intercambios entre los integrantes, de información sobre la enfermedad y tratamiento, y de seguridad e inserción de la familia en el tratamiento.

Consideraciones finales: El grupo de apoyo puede ser entendido como una acción estratégica de cuidado al familiar, repercutiendo en su vida y en el tratamiento de quien está internado.

Palabras clave: Enfermería. Salud mental. Servicios de salud. Grupos de autoayuda. 


\section{—INTRODUÇÃO}

A Reforma Psiquiátrica consistiu em um longo e complexo processo de reorientação do modelo assistencial em saúde mental, que questionou o modelo asilar baseado no hospital psiquiátrico, defendendo o cuidado no território, a reinserção social e a manutenção dos vínculos familiares ${ }^{(1-2)}$.

Nesse sentido, a família, nos dias atuais, em face das políticas públicas brasileiras de saúde mental, que priorizam a desospitalização e a desisntitucionalização da assistência, tem muita importância para o tratamento ${ }^{(3)}$. Desse modo, não se pode separar a doença do contexto familiar e, por ser um elemento tão essencial, a família deve ser compreendida como uma aliada da equipe de saúde, atuando como um recurso na promoção do conforto, para o paciente adquirir confiança e, assim, investir em seu tratamento(4).

No entanto, além de perceber a família como grande aliada, é necessário enxergá-la como uma unidade que necessita de cuidado por parte dos profissionais de saúde ${ }^{(5)}$. Assim, os serviços de saúde devem considerar a família como potencial usuária, tendo em vista suas dificuldades no dia a dia e a sobrecarga no acompanhamento do processo terapêutico, e ao mesmo tempo instrumentalizá-la para compartilhar o cuidado de seu familiar necessitado de cuidados(6).

Nessa perspectiva, o trabalho com grupos de apoio destinado aos familiares torna-se fundamental no percurso da desinstitucionalização, sendo uma importante estratégia de atendimento das necessidades dos familiares que certamente refletirá no cuidado no domicílio à pessoa com sofrimento psíquico ${ }^{(7)}$. Dessa maneira, o tratamento em que a família é cuidada demonstra a influência positiva que o meio familiar pode exercer no curso do tratamento dos vários transtornos psiquiátricos ${ }^{(8)}$.

Em relação a esse cuidado ao familiar, o grupo de apoio aos familiares emerge como um espaço que permite não apenas a apropriação de saberes e fazeres relacionados aos modos dos familiares de conviverem com o sofrimento psíquico, mas, sobretudo, como um espaço de construção permanente de novos sentidos possíveis sobre o processo saúde/doença e de novas redes de apoio social(9-10).

O interesse em desenvolver o tema surgiu no decorrer do trabalho na residência multiprofissional em saúde, no qual se observou a importância do grupo de apoio realizado em uma unidade de internação psiquiátrica para os familiares de pessoas com transtornos mentais, instigando na busca por analisar a percepção desses familiares sobre o grupo desenvolvido a partir dos pressupostos da Reforma Psiquiátrica.

Assim, por entender a família como essencial no tratamento da pessoa com sofrimento psíquico e os grupos de apoio como estratégias potentes de cuidado a esses fami- liares, questiona-se: Qual é a percepção dos familiares de pessoas com transtornos mentais sobre o grupo de apoio desenvolvido em uma unidade de internação psiquiátrica?

Tem-se por objetivo analisar a percepção de familiares de pacientes com sofrimento psíquico sobre o grupo de apoio desenvolvido em uma unidade de internação psiquiátrica de um hospital geral.

A contribuição deste trabalho é de fundamentar o processo de constituição de grupos de apoio aos familiares e perceber a nova realidade exercida nos serviços de saúde, em especial, a unidade de internação psiquiátrica, na perspectiva da atual legislação de saúde mental. Além disso, pretende-se proporcionar subsídios para a construção do conhecimento sobre o tema, estimulando a formulação de novos modos de inserir os familiares no tratamento a partir das suas necessidades de cuidado nas instituições de saúde mental.

\section{— MÉTODO}

O presente estudo é uma pesquisa exploratória, descritiva, com abordagem qualitativa(11). O estudo foi desenvolvido em uma unidade de internação psiquiátrica (UIP) de um hospital geral do Sul do Brasil. Esta unidade conta com 36 leitos (26 leitos SUS e 10 leitos de convênio) e atende situações psiquiátricas agudas graves. Conta com uma equipe multiprofissional composta por médicos, enfermeiros, técnicos de enfermagem, residentes, psicólogos, nutricionistas, assistente social e terapeuta ocupacional.

O grupo de apoio desenvolvido na UIP é aberto aos familiares e acompanhantes dos pacientes internados. Não tem um número pré-definido de vagas e todos os familiares dos pacientes são convidados a participar. A frequência é semanal, com uma hora de duração, e ocorre em uma sala da própria unidade. O grupo é coordenado por uma enfermeira e um psicólogo e conta com uma participação média de oito a dez familiares em cada encontro.

Este grupo tem por objetivo a promoção de um espaço de conversação e apoio para o enfrentamento da doença e a compreensão dos fatores emocionais envolvidos, além de fomentar a reflexão e a troca de experiências entre pessoas que vivenciam a situação de ter um familiar com sofrimento psíquico.

Os participantes da pesquisa foram familiares de pacientes internados da UIP. Teve como critérios de inclusão estar participando do grupo no momento da coleta de dados, ter frequentado o grupo no mínimo uma vez, ter mais de 18 anos, demonstrar boa comunicação e não apresentar déficit cognitivo.

Por se tratar de uma pesquisa qualitativa, o número total de sujeitos entrevistados foi definido pelo critério de 
saturação dos dados, que é um conceito amplamente utilizado em pesquisas qualitativas na área da saúde ${ }^{(12)}$. Dessa maneira, foram entrevistados dez familiares.

Os dados foram coletados no mês de junho de 2016 através de uma entrevista semiestruturada sobre a percepção dos familiares acerca do grupo de apoio, a saber: "Qual é a sua percepção do Grupo de Apoio?", "O grupo contribui em algum aspecto em relação ao seu familiar? Qual?", "Como você se sente após participar do grupo?".

Os familiares foram convidados a participar do estudo após o término do grupo, sendo agendados dia e hora para a coleta dos dados conforme disponibilidade dos participantes. As entrevistas tiveram duração em média de 15 minutos e se realizaram em um consultório do UIP, gravadas e posteriormente transcritas para análise.

A análise temática dos dados se deu segundo Minayo(11), sendo esta técnica dividida em três etapas: na pré-análise realizaram-se a transcrição literal das entrevistas e a elaboração das unidades de registro; na fase de exploração do material, os dados foram codificados e agrupados por semelhanças e diferenças, gerando categorias e subcategorias; e, na fase de tratamento dos resultados obtidos e interpretação, selecionaram-se as falas mais significativas, que foram discutidas a partir de estudiosos da temática, emergindo uma categoria: "A percepção dos familiares sobre o grupo de apoio".

A pesquisa foi aprovada pelo Comitê de Ética do referido hospital, conforme o Protocolo 1.424.320, sendo considerados os aspectos éticos envolvendo seres humanos ${ }^{(13)}$. Os participantes assinaram o Termo de Consentimento Livre e Esclarecido após terem sido informados sobre os objetivos da pesquisa. As entrevistas foram codificadas pela letra E seguida pelo número da ordem cronológica em que das entrevistas, como, por exemplo, E1, que se refere ao primeiro entrevistado, preservando o anonimato dos entrevistados.

\section{口 RESULTADOS}

\section{A percepção dos familiares sobre o grupo de apoio}

Dos familiares entrevistados, quatro eram mães, três eram esposas, dois, filhos e uma, irmã. Apenas um entrevistado era do sexo masculino. A faixa etária variou entre os 35 anos e 63 anos, com maior concentração na faixa dos 50 anos. No momento da coleta de dados, quatro entrevistados exerciam o trabalho informal, dois estavam desempregados, dois faziam atividades do lar e uma estava afastada. Em relação ao seu familiar internado na UIP, quatro haviam se internado por depressão, quatro, por esquizofrenia e dois, por uso de drogas.
Os familiares entrevistados reconheceram o grupo como um espaço de apoio mútuo e de fortalecimento, pois percebiam que não eram os únicos a enfrentar a experiência de ter um familiar internado em uma UIP. Sentiam-se fortalecidos a partir das experiências dos outros, conforme pode ser evidenciado nas seguintes falas:

[...] para mim estava num poço que eu não conseguia sair mais de dentro, e depois, com o grupo, eu passei a ver que não era assim. Comecei a entender que eu não sou a primeira que está passando por isso, e que ela vai ter esses altos e baixos, e eu vou ter que estar sempre firme pra conseguir levantar ela (E4).

Bem dificil, a familia toda sofre. Mas o grupo ajuda bastante, ajuda a te fortalecer, porque às vezes a gente perde as forças, eu acho muito importante, para mim ajudou bastante, porque não é fácil (E10).

Os familiares que cuidam de pacientes com sofrimento psíquico necessitam de apoio, pois frequentemente sua saúde mental também pode tornar-se prejudicada, porque são afetados com as mudanças no seu cotidiano. Além da falta de preparo e de conhecimento para lidar com esta situação, convivem diariamente com o estigma ea discriminação da sociedade, o que acarreta num ônus significativo em suas vidas.

Nesse sentido, o grupo pode contribuir para mudanças no comportamento que possibilitam melhora no convívio e redução de conflitos.

Os membros do grupo atuam na ressignificação da percepção dos familiares de não estarem desamparados, ao reconhecerem que os problemas vividos com a pessoa em sofrimento psíquico são comuns a todos, gerando espaço de escuta e trocas entre os integrantes.

Auxilia muito, é muito bom porque a gente ouve o que o grupo está dizendo, sempre tem uma opinião diversa, um fala uma coisa, outro fala outra coisa [...], cada um dá a sua opinião, e a coordenadora aceita a opinião de todos e cada um contribui um com o outro (E1).

Eu acho que o grupo é muito bom porque as pessoas têm a chance de poder expor seus problemas e um ajudar o outro (E2).

O grupo ajudou porque a gente troca experiência, as pessoas trocam como agem, o que sentem. Você começa a ouvir o que o pessoal fala, do problema de cada um, daí você aprende como tem que agir, fazer, a ter mais paciência e compreensão (E6). 
A importância da troca de experiências cotidianas com os familiares é propícia para a coesão grupal, possibilitando a expressão de temas que interessem a todos. Pode-se intensificar esse sentimento, enfatizando as semelhanças existentes entre as experiências, assim, quando interagem um com o outro, os membros do grupo podem oferecer suporte, compreensão, conselhos e encorajamento.

Os familiares entrevistados relataram uma necessidade de ter mais orientação em relação à crise, de conhecer a patologia (diagnóstico, causas, tratamento e prognóstico) e aprender sobre como abordar seu familiar em momentos de crise. Dessa maneira, os familiares identificaram que o grupo ajuda no reconhecimento das dificuldades vivenciadas no cotidiano com a pessoa com sofrimento psíquico, ofertando aos familiares um espaço de auxílio de informações sobre a doença e o tratamento.

Eu achei bem importante porque esclarece bastante coisa para gente. Inclusive eu estava com bastante dúvidas quando a R. fosse para casa. Até eu disse para o pessoal do grupo que eu era marinheira de primeira viagem, eque precisava muito de algum esclarecimento porque eu não sabia, estava totalmente perdida. Mas a profissional e os outros familiares que estavam ali disseram mais ou menos como poderia ser, então o grupo foi muito importante (E4).

Então, para mim foi muito importante, me ajudou bastante, eu comecei a ficar melhor até para poder ajudar, entender a doença mais (E5).

Ah, me ajudou bastante, deixou eu entender várias coisas que o W. faz, por mais anos que eu lide com ele, muita coisas eu não entendia. Daí eu perguntava, e eles me explicaram, e me apoiaram em muitas coisas que o W. faz, que eu tenho que entender para lidar melhor com ele. Me auxiliou para me ajudar a lidar melhor com ele (E10).

O grupo de apoio estudado permitiu um trabalho colaborativo entre familiares e profissionais, compartilhando informações sobre a doença e discutindo conjuntamente as dúvidas e dificuldades sobre o tratamento. Assim, a sobrecarga resultante dos primeiros episódios agudos da doença tende a diminuir, na medida em que o familiar adquire mais informações e experiência para lidar com os problemas.

Cabe ao coordenador do grupo criar situações propícias para o aprendizado conjunto acerca da doença e das formas de lidar com as dificuldades enfrentadas no cotidiano por essas famílias.

Através da fala dos entrevistados identifica-se que o modo de se relacionar com os familiares constitui-se em um importante desafio para os cuidadores, que podem apresentar mais dificuldade em compreender as formas de agir, o que resulta em dificuldades de aceitação ou até mesmo culpabilização. O grupo de apoio foi entendido pelos entrevistados como um espaço que auxilia no processo de aceitação da doença.

Eu não me senti tão sozinha, e vi que existem outras pessoas que te entendem, mesmo que talvez não seja a mesma situação. Isso me auxiliou. Eu fiquei mais segura, e vi que ele está sendo bem assistido aqui dentro, daí eu fiquei mais tranquila e aprendi a aceitar melhor a doença e como ele étambém (E3).

No início a gente não aceita. Na verdade, a gente nunca vai aceitar, a gente quer que sempre tenha uma melhora, tem a esperança, só que, no caso de algumas doenças, não é isso o que acontece [...] mas eu aprendi a aceitar um pouco mais, lidar mais com isso. Pra mim o grupo contribuiu bastante, não que eu hoje eu aceite, a gente nunca aceita, mas a gente aprende a lidar (E5).

Através da participação nos grupos, o entendimento sobre a doença facilitou o processo de aceitação da doença pelos familiares, possibilitando a busca por melhores formas de lidar com a pessoa que vive com sofrimento psíquico. Dessa maneira, os entrevistados valorizam a oportunidade de conversar no grupo sobre seus medos, receios e dificuldades, facilitando o processo de aceitação na medida em que possuem mais informações sobre a doença e tratamento.

A insegurança e o medo também eram sentimentos frequentes entre os familiares entrevistados, que enfatizaram os benefícios de ter um espaço para aliviar as tensões, onde pudessem ser escutados e cuidados, sentindo-se seguros e mais tranquilos com a internação e o processo de adoecer do seu familiar.

Eu me senti mais segura, para passar mais segurança para ela [paciente]. Eu acho que só no eu conversar com ela, e falar pra ela que não é a única, que tem pessoas que já tiveram internações em muitos outros hospitais, e aqui ela está, pelo o que o pessoal comentou, no melhor lugar, eu acho que isso ajudou bastante ela também. Porque ela estava muito deprimida, e hoje ela já está bem mais animada. Eu fui pra casa bem mais tranquila, vendo o problema dela não é o fim do mundo (E4).

Acho que foi bem produtivo assim, as reuniões, no meu caso eu não consegui vir em todas, mas as que eu vim me 
auxiliaram bastante, eu saía daqui mais calma, mais tranquila, coloquei para fora o que eu estava sentindo sobre a doença dela (E5).

Os entrevistados perceberam que no espaço do grupo sentiam que não estavam sozinhos, que tem uma equipe à qual poderiam recorrer quando necessitassem e outros familiares na mesma situação. Essa condição proporciona aos familiares sentimentos de segurança e tranquilidade, repercutindo diretamente na relação entre a família e a pessoa com sofrimento psíquico.

A família, ao ser reconhecida como protagonista no tratamento do seu familiar internado na UIP, deve ser considerada pelos profissionais em suas especificidades, opiniões e ações. Nessa perspectiva, o cuidado à família como unidade de cuidado torna-se fundamental no tratamento e o grupo surge como uma potente estratégia de apoio na perspectiva dos próprios entrevistados.

Para mim o grupo de apoio para a família é muito importante porque a base é a família. O paciente está doente e, a família, ela acaba ficando junto doente, então não adianta tratar o paciente e a família não, porque a família vai continuar doente, e essa família doente não vai poder ajudar na melhora (E5).

Eu percebi, aqui dentro, que a ajuda da família é importante. Muito importante, pois você vai vendo que a família é ajudada aqui e depois ela ajuda o paciente lá fora (E6).

O grupo de apoio é uma estratégia importante de cuidado aos familiares de pessoas com transtornos mentais na UIP, que modifica a lógica de culpabilização que por muitos anos perpetuou na saúde mental, para uma lógica da família protagonista no processo de reabilitação do seu familiar. Assim, a percepção dos familiares revela que as preocupações advindas do cuidado podem impactar negativamente na reabilitação da pessoa internada na UIP e que o grupo de apoio é um espaço potente para o cuidado das famílias, repercutindo positivamente no tratamento.

\section{DISCUSSÕES}

A Reforma Psiquiátrica brasileira propiciou um repensar sobre os saberes e as práticas dos profissionais de saúde que atuam nos serviços que compõem a rede de saúde mental. Essa reflexão possibilitou que os profissionais vislumbrassem outras formas de cuidar das pessoas com transtornos mentais, entendendo a família como fundamental nesse processo ${ }^{(1)}$.
Assim, espaços como os grupos de apoio têm sido importantes no contexto dos serviços de saúde que atuam na perspectiva da Atenção Psicossocial, uma vez que os familiares sentem-se mais fortalecidos, tranquilos e seguros em relação ao tratamento e o processo de reabilitação do seu paciente internado quando participam desses espaços ${ }^{(2)}$.

As famílias percebem o grupo como um espaço que Ihes proporciona a possibilidade de expressar suas angústias e medos, que em outros contextos de vida não teriam, e que isto também é facilitado pela identificação mútua que estabelecem entre os integrantes. Assim, o potencial preventivo desses grupos emerge da possiblidade de pessoas que vivenciam situações semelhantes exporem seus conhecimentos e suas vivências por meio de depoimentos e troca de experiências ${ }^{(5)}$.

A participação nos grupos favorece a troca de experiências, o enfrentamento dos problemas, gerando apoio mútuo e solidariedade, além de promover um sentimento de pertencimento e confiança, o que fortalece e favorece positivamente o resgate da autoestima. Ouvir sobre os problemas dos outros e não se sentir como o único propicia o sentimento de universalidade ${ }^{(14)}$.

A tarefa de cuidar e viver com alguém com sofrimento psíquico pode ser muito desgastante, principalmente nos momentos de crise, em que se faz necessária capacitação dos familiares e cuidadores em relação ao manejo desses momentos críticos. Assim, os grupos de apoio podem atuar como espaços de educação em saúde ou psicoeducacionais, propiciando a estas famílias formas de manejo, assim como um espaço para externarem sentimentos dos encargos subjetivos e objetivos que a convivência com o doente acarreta ${ }^{(1)}$.

Dessa maneira, os familiares buscam informações sobre a doença e sobre o tratamento, o que colabora para meIhorar a estabilidade emocional dentro do núcleo familiar, permitindo que a família eleve sua capacidade e habilidade para cuidar. No entanto, a falta de orientação pode gerar conflitos entre o paciente e seus familiares, refletindo negativamente sobre o processo terapêutico, e acarretando aumento da sobrecarga familiar(15-16).

O contato com pessoas que também estejam passando por situações semelhantes ameniza o sofrimento, reduz o isolamento, possibilitando trocas de experiências e, consequentemente, maior apoio e conforto(1). Através dos grupos, os familiares compreenderam melhor a doença e tratamento, havendo melhora significativa no relacionamento do paciente com a família, diminuição de ansiedade e agressividade, bem como(,) aumento da tolerância e força vontade ${ }^{(17-18)}$.

Quando um membro da família adoece mentalmente, a família enfrenta preconceitos sociais e carrega consigo 
estigmas que ela própria sustenta, pois, ao se depararem com essa realidade, muitos acreditam que a melhor solução é o isolamento. Todas as modificações e situações vivenciadas devido à doença causam sofrimento à família, principalmente no período inicial e para familiares que estão vivenciando a crise pela primeira vez ${ }^{(19)}$. Na fase de adaptação a família muda sua rotina de vida, e com essa mudança verifica-se a falta de preparo para lidar com as novas situações, e com isso o grupo de apoio torna-se muito importante nesse processo ${ }^{(8)}$.

Ressalta-se, diante das questões especialmente apontadas sobre as dificuldades de cuidar e o sofrimento decorrente, a necessidade de intervenções terapêuticas voltadas para a saúde do cuidador, para que esse possa atenuar seu sofrimento e repensar sua vida ocupacional, além de aprender estratégias adequadas ao cuidado do familiar ${ }^{(20)}$.

Neste estudo, entende-se que isso significa ter uma nova lógica de atendimento à pessoa com sofrimento psíquico, a da família não só ser incluída no plano assistencial, mas também constituir-se como foco do cuidado. Isto, porém implica uma assistência implementada de acordo com as necessidades de cada família, levando-se em consideração sua unicidade e realidade. Cabe aos profissionais dos serviços de saúde mental estarem atentos às demandas e às particularidades de cada unidade de cuidado, desenvolvendo estratégias e criando espaços que contemplem a multiplicidade de necessidades que possam vir a se apresentar ${ }^{(20)}$.

Os coordenadores dos grupos precisam trabalhar a aceitação junto ao familiar para que esse processo se dê da forma menos sofrida possível(15). O tratamento de um paciente com sofrimento psíquico está intimamente atrelado ao envolvimento da família, pois na maioria das vezes esta faz parte da base do indivíduo. Sendo assim, a evolução do tratamento depende muito da aceitação e apoio da família e da inclusão social do mesmo, pois, somente com o sentimento de pertencimento e autonomia, o ser humano poderá resgatar sua autoestima, autoimagem e estabelecer-se como sujeito de sua própria história(20).

A não aceitação da doença por parte dos familiares pode gerar um sentimento como se nunca estivessem totalmente tranquilos, devido a muitas preocupações, principalmente na supervisão de comportamentos que o paciente adota ou pode adotar, como tentativas de fuga, suicídio e na realização de atividades diárias(15).

O grupo de apoio demonstra que se faz necessária a implantação de estratégias que tenham como foco os cuidados aos familiares que cuidam de pessoas com transtornos mentais graves e persistentes, de modo a lhes oportunizar a reflexão compartilhada sobre os possíveis modos de cuidar de si e do outro, Ihes proporcionando segurança e tranquili- dade ${ }^{(10)}$. Dessa maneira, o grupo de apoio pode ser entendido uma estratégia de intervenção e cuidado, instrumentalizando o familiar para um cuidado eficaz de si e da pessoa com sofrimento psíquico, com a finalidade de diminuir a sobrecarga por que passam no exercício de suas tarefas de cuidar e para que possam ressignificar suas experiências ${ }^{(20)}$.

É nesse contexto que o enfermeiro, seja como integrante da equipe de saúde, seja como coordenador dos grupos de apoio, pode contribuir para a inserção dos familiares nesses espaços, utilizando a escuta, empatia, acolhimento, vínculo e o trabalho em equipe multiprofissional como instrumentos de trabalho no seu cotidiano. Assim, poderá ser capaz de suprir as demandas e necessidades apontadas pelos familiares, além de diminuir encaminhamentos desnecessários e o tempo de espera por consultas especializadas ${ }^{(17)}$.

Os participantes deste estudo perceberam o grupo de apoio como indispensável para que se consolide um cuidado efetivo à pessoa com sofrimento psíquico e sua família no paradigma da desinstitucionalização e da atenção psicossocial. Sair da lógica curativista, baseada apenas nos sinais e sintomas e no atendimento por consultas, é repensar as práticas dos serviços de saúde que constituem a rede ${ }^{(17)}$.

Os serviços de saúde mental que constituem a rede devem propiciar às pessoas doentes e seus familiares o fortalecimento das relações no seu meio social e o desenvolvimento de diversas possibilidades de trocas sociais, indo além do ambiente familiar ou domicilio, estendendo-se para diversos espaços na sociedade, o que pode contribuir para o processo de reabilitação e inclusão social dos sujeitos em sofrimento psíquico (16). Dessa forma, prima-se por um tratamento onde a família seja valorizada, esteja orientada e participe efetivamente das ações promovidas.

\section{— CONSIDERAÇÕES FINAIS}

Este estudo teve por objetivo analisar as percepções dos familiares de pessoas com transtornos mentais sobre o grupo de apoio desenvolvido em uma unidade de internação psiquiátrica.

Através da análise dos resultados foi possível compreender que os entrevistados perceberam o grupo como um espaço de apoio mútuo e de fortalecimento; espaço de escuta e trocas entre os integrantes; espaço de auxílio de informações sobre a doença e o tratamento; espaço que auxilia no processo de aceitação da doença, proporcionando segurança e tranquilidade; e o grupo de apoio como uma potente estratégia de apoio na perspectiva da Reforma Psiquiátrica e da Atenção Psicossocial.

Diante da importância que os familiares possuem no tratamento da pessoa com sofrimento psíquico, identi- 
ficamos neste estudo que o grupo de apoio é uma ação estratégica de cuidado que ajuda o familiar a reduzir sua sobrecarga emocional, permitindo que este consiga exercer o cuidado com um desgaste menor, repercutindo na sua vida e no tratamento de quem está internado.

Sendo assim, ressaltamos a relevância deste estudo, pois os resultados contribuem para o fortalecimento dessa estratégia de cuidado no âmbito da UIP e que podem ser utilizados em outros serviços de saúde mental. Além disso, os resultados desta pesquisa podem incentivar os profissionais da equipe multiprofissional, dentre eles, o enfermeiro, na coordenação dos grupos de apoio e na divulgação desses espaços, favorecendo a concepção de não culpabilização, de espaço de cuidado aos familiares e no combate aos preconceitos e discriminação.

No entanto, destaca-se que este estudo teve limitações, como: um número pequeno de entrevistados e a pesquisa ter sido desenvolvida em apenas um grupo de apoio e em uma determinada UIP. Assim sendo, sugere-se que futuros estudos abordem mais amplamente tais aspectos tão importantes na área de saúde mental.

Conclui-se que o entendimento da família como coparticipante do processo terapêutico pode influenciar a busca de novas possibilidades de atendimento e de mecanismos que propiciem a autonomia do usuário, a conquista de direitos e o exercício da cidadania.

\section{口 REFERÊNCIAS}

1. Bessa J, Waidman M. Family of people with a mental disorder and needs in psychiatric care. Texto Contexto Enferm. 2013;22(1):61-70. doi: http://dx.doi. org/10.1590/50104-07072013000100008.

2. Ministério da Saúde (BR). Política Nacional de Saúde Mental. Braślia (DF); 2001 [citado 2015 out 11]. Disponivel em: http://www.cress-pa.org.br/files/ u4/11_Pol_SaudeMental.pdf.

3. Maciel SC. Reforma psiquíátrica no Brasil: algumas reflexões. Cad Bras Saúde Mental. 2012 [citado 2016 jan 12];4(8):73-82. Disponível em: http://stat.cbsm. incubadora.ufsc.br/index.php/cbsm/article/view/2021/2307.

4. Borba L, Guimarães AN, Mazza VA, Maftum MA. Mental health care based on the psychosocial model: reports of relatives and persons with mental disorders. Rev Esc Enferm USP. 2012;46(6):1406-14. doi: http://dx.doi.org/10.1590/ S0080-62342012000600018.

5. Cid M, Pereira L. Adolescentes com dificuldades relacionadas à saúde mental, moradores de áreas rurais: percepções sobre família, escola e contexto de moradia. Cad Ter Ocup UFSCar. 2016;24(3):543-55. doi: http://dx.doi. org/10.4322\%2F0104-4931.ctoA00724.
6. Duarte MLC, Vianna KR, Olschowsky A. Crack users' evaluation regarding family groups in the Psychosocial care center. Cogitare Enferm. 2015;20(1):81-8. doi: http://dx.doi.org/10.5380/ce.v20i1.37597.

7. Ferro LF. Grupo de convivência em saúde mental: intersetorialidade e trabalho em rede. Cad Ter Ocup UFSCar. 2012;23(3):485-98. doi: http://dx.doi. org/10.4322\%2F0104-4931.ctoA00558.

8. Nolasco M, Bandeira M, Oliveira MS, Vidal CEL. Family caregivers' burden as a function of psychiatric patients diagnostics. J Bras Psiquiatr. 2014;63(2):89-97. doi: http://dx.doi.org/10.1590/0047-2085000000011. Portuguese.

9. Duarte MLC, Thomas J, Olschowsky A. Care for mental health in pychiatric hospitalization: the perception of the family members. Cogitare Enferm. 2014;19(1):131-7. doi: http://dx.doi.org/10.5380/ce.v19i1.35969.

10. Costa L, Ribeiro A, Junqueira EL, Meneses FFF, Stroher LMC. Grupo multifamiliar com adolescentes ofensores sexuais. Psico. 2011 [citado 2016 jan 20];42(4):450-6. Disponível em: http://revistaseletronicas.pucrs.br/ojs/index. php/revistapsico/article/view/10729/7448.

11. Minayo M. Pesquisa social: teoria, método e criatividade. 29. ed. Petrópolis: Vozes; 2010.

12. Pope C, Mays N. Pesquisa qualitativa na atenção à saúde. 2. ed. Porto Alegre: Artmed; 2005.

13. Ministério da Saúde (BR). Conselho Nacional de Saúde. Resolução nº 466, de 12 de dezembro de 2012. Aprova as diretrizes e normas regulamentadoras de pesquisas envolvendo seres humanos. Diário Oficial da União [da] República Federativa do Brasil. 2013 jun 13;150(112 Seção 1):59-62.

14. Silva TFC. Assessing fidelity in psychosocial interventions: a systematic literature review. J Bras Psiquiatr. 2014;63(3):260-71. doi: http://dx.doi. org/10.1590/0047-2085000000034. Portuguese.

15. Alves DR, Morais TTM, Rocha SP, Rocha NNV, Duarte SR, Sampaio FFF. Grupo de familiares em CAPS AD: acolhendo e reduzindo tensões. Sanare. 2015 [citado 2016 fev 10];14(1):81-6. Disponível em: https://sanare.emnuvens.com.br/sanare/article/view/613/330.

16. Queirós PS, Pires LM, Matos MA, Junqueira ALN, Medeiros M, Souza MM. Conceptions of parents of adolescent students about the sexuality of their children. Rev Rene. 2016 [citado 2017 jan 05];17(2):293-300. Disponível em: http:// www.periodicos.ufc.br/index.php/rene/article/view/3044/2347.

17. AlvarezS, Gomes GC, Oliveira AMN, Xavier DM. Support group as a strategy of care: the importance for relatives of drug users. Rev Gaúcha Enferm. 2012;33(2):1028. doi: http://dx.doi.org/10.1590/S1983-14472012000200015. Portuguese.

18. Menezes SL, Souza MCBM. Psychoeducational group and bipolar affective disorder: a reflection on the asylum and psychosocial models. Rev Esc Enferm USP. 2011;45(4):996-1001. doi: http://dx.doi.org/10.1590/S008062342011000400029

19. Wesner AC, Gomes J, Silva TD, Blaya C, Manfro GG, Heldt E. Effect of cognitive-behavioral group therapy for panic disorder in changing coping strategies. Compr Psychiatry. 2014:55(1):87-92. doi: https://doi.org/10.1016/j. comppsych.2013.06.008.

20. Behenck A, Wesner AC, Finkler DC, Heldt E. Contribution of Group Therapeutic Factors to the Outcome of Cognitive-Behavioral Therapy for Patients with Panic Disorder. Arch Psychiatr Nurs. 2017;31(2):142-6. doi: http://doi.org/10.1016/j. apnu.2016.09.001.

Recebido: 14.06.2017

Aprovado: 17.11.2017 